\title{
Extraction of the respiratory signal from small-animal CT projections for a retrospective gating method
}

\author{
C Chavarrías, J J Vaquero, A Sisniega, A Rodríguez-Ruano, \\ M L Soto-Montenegro, P García-Barreno and M Desco
}

Unidad de Medicina y Cirugía Experimental, Hospital General Universitario Gregorio Marañón, Anexo Psiquiatría, 1 Planta. C/ Ibiza, 43. Madrid 28007, Spain

\begin{abstract}
We propose a retrospective respiratory gating algorithm to generate dynamic CT studies. To this end, we compared three different methods of extracting the respiratory signal from the projections of small-animal cone-beam computed tomography (CBCT) scanners. Given a set of frames acquired from a certain axial angle, subtraction of their average image from each individual frame produces a set of difference images. Pixels in these images have positive or negative values (according to the respiratory phase) in those areas where there is lung movement. The respiratory signals were extracted by analysing the shape of the histogram of these difference images: we calculated the first four central and non-central moments. However, only odd-order moments produced the desired breathing signal, as the even-order moments lacked information about the phase. Each of these curves was compared to a reference signal recorded by means of a pneumatic pillow. Given the similar correlation coefficients yielded by all of them, we selected the mean to implement our retrospective protocol. Respiratory phase bins were separated, reconstructed independently and included in a dynamic sequence, suitable for cine playback. We validated our method in five adult rat studies by comparing profiles drawn across the diaphragm dome, with and without retrospective respiratory gating. Results showed a sharper transition in the gated reconstruction, with an average slope improvement of $60.7 \%$.
\end{abstract}

\section{Introduction}

Computed tomography (CT) has been widely applied in the diagnosis and treatment planning of many diseases because of the precise non-invasive morphological information that it provides (Jose et al 1983, Mira et al 1982). In particular, the utility and relevance of animal models of pulmonary diseases have already been proved (Hardouin and Nagy 2000). However, imaging 
the thorax and abdomen in vivo is limited by respiratory motion, which causes blurring and artefacts in the CT projections and in the reconstructed tomographic images.

Despite the similarities in respiratory motion between humans and small laboratory animals, different processing approaches are advisable due to differences in size, anatomy, and the different CT geometries and technologies used. The complexity of respiratory blurring reduction techniques increases whenever the field of view (FOV) does not fully cover the subject scanned. In this case, it becomes necessary either to acquire images at different bed positions with step-and-shoot protocols (Rietzel et al 2005, Simon et al 2005, Lu et al 2005b, Handels et al 2006, Ehrhardt et al 2007, McClelland et al 2006) or to move the sample continuously under the FOV (Keall et al 2004, Vedam et al 2003, Ford et al 2003). For small rodent lung imaging this is not necessary, as the thorax is completely covered by the FOV of CBCT scanners. Thus, a synchronization of the frames acquired at different axial angles around the animal will suffice. Most of the literature on respiratory motion compensation focuses on human imaging.

The different methods proposed in the literature to compensate lung and rib motion can be described as prospective or retrospective, depending on knowledge of the respiratory phase when data are acquired. In prospective techniques, acquisition is synchronized with the patient's breathing, and all the projections are acquired during the same respiratory phase by means of a trigger produced by real-time tracking of the respiratory signal (Badea et al 2004, Ford et al 2005). Several types of devices have been used to generate an external respiratory signal: reflective markers attached to the subject's body surface which enable the tracking of the antero-posterior thorax thickness via video analysis (Pan et al 2004, Keall et al 2004, Rietzel et al 2005, Underberg et al 2004, Vedam et al 2003), spirometers to directly measure the air volume exchanged with the atmosphere (Simon et al 2005, Low et al 2003), thermometers placed in the patient's nostrils (Damen et al 2004) and pressure transducers placed under the body or inside an elastic belt (Drangova et al 2007, Ford et al 2005, 2007, Kleshneva et al 2006). All of them have been successfully used in gating systems, but whereas spirometers yield very accurate results (Lu et al 2005a, 2005b, Low et al 2003), there is no agreement in the literature on using reflective markers on the chest (Koch et al 2004, Beddar et al 2007). The result of any prospective protocol is a single 3D image obtained during the specific respiratory phase to which the scanner was gated. Whenever a complete dynamic study is desired, several acquisitions are necessary, thus increasing imaging time.

On the other hand, retrospective algorithms (Damen et al 2004, Drangova et al 2007, Ehrhardt et al 2007, Ford et al 2007, Guerrero et al 2006, Handels et al 2006, Hu et al 2004, Keall et al 2004, Kleshneva et al 2006, Li et al 2006, Li and Xing 2007, Low et al 2003, McClelland et al 2006, Pan et al 2004, Rietzel and Chen 2006, Rietzel et al 2005, Schreibmann et al 2006, Simon et al 2005, Sonke et al 2005, Underberg et al 2004, Vedam et al 2003, Xu et al 2006, Zeng et al 2005) do not require any trigger signal during the acquisition, although they are constrained by the need to acquire at least one complete respiratory cycle per slice to avoid any empty phase bins. Therefore, the acquisition protocol usually requires multiple frames from every projection angle, each one corresponding to a different point during the breathing cycle. Two strategies have been proposed to establish the correspondence between projection frames and their respective respiratory phases: (a) sorting the images into a different number of phase bins (Damen et al 2004, Drangova et al 2007, Ford et al 2007, Hu et al 2004, Keall et al 2004, Kleshneva et al 2006, Li et al 2006, Li and Xing 2007, Pan et al 2004, Rietzel and Chen 2006, Rietzel et al 2005, Simon et al 2005, Sonke et al 2005, Underberg et al 2004, Vedam et al 2003) and (b) applying registration techniques to compensate the elastic motion of lungs (Zeng et al 2005, Xu et al 2006, Schreibmann et al 2006, McClelland et al 2006, Handels et al 2006, Guerrero et al 2006, Ehrhardt et al 2007). The latter approach is usually 
accomplished by deforming and merging the different volumes from each respiratory phase into a single reconstructed image. In order to assign the appropriate bin to the different CT projections, some authors (Drangova et al 2007, Ford et al 2007, Keall et al 2004, Kleshneva et al 2006, Li et al 2006, Li and Xing 2007, Low et al 2003, Pan et al 2004, Rietzel and Chen 2006, Rietzel et al 2005, Simon et al 2005, Underberg et al 2004, Vedam et al 2003) used an external signal obtained during the acquisition, as in the case of prospective triggering, whereas others (Sonke et al 2005, Rit et al 2005, Hu et al 2004) extracted the gating reference directly from the projections by means of image processing techniques.

Extensive research has been conducted to automatically extract the respiratory gating signal from the projection images before the data are reconstructed into three dimensions. Tanaka et al proposed four methods for conventional (non-CT) coronal chest radiographs: two delimited the lungs by detecting the tissue-air transition, another was based on the correlation of the image with a reference radiograph taken at breath-hold and the fourth tracked the trajectories of a set of points placed near the diaphragm (Tanaka et al 2006). Zijp et al (2004) detected the diaphragm transition by projecting a threshold mask in the cranio-caudal axis and obtained the respiratory signal from its movement. Rit et al (2005) developed a block matching algorithm (BMA) similar to that of Tanaka's fourth method to track points in the lungs. Hu et al (2004) extracted the respiratory signal by adding the pixel values of a lung region of interest (ROI). These three methods were also successfully applied to CT scans (Sonke et al 2005, Rit et al 2005, Hu et al 2004), which require more robust and stable processing techniques than coronal radiographs to extract the breathing signal.

The evolution of 4D-CT (respiratory-gated CT) led to the use of image registration techniques that allegedly provide higher resolution and smoother transitions than phase-sorting methods (Xu et al 2006). Breath-hold has also been combined with registration methods; a scan acquired at breath-hold was usually taken as a static reference to characterize the deformation in subsequent acquisitions. Ideally, during a breath-hold, the thorax is supposed to be almost static and blurring should be minimal. Although breath-hold has been widely used in the literature, some conditions may prevent patients from cooperating and, therefore, research has focused on free-breathing techniques. The deformation field obtained from the registration process can directly indicate the respiratory phase, making respiratory signal extraction unnecessary. Some authors registered segmented CT axial slices to a breath-hold reference volume (Xu et al 2006), others registered reconstructed volumes to a breath-hold reference volume, until their respective projections were as similar as possible (Zeng et al 2005). However, the most common approach involves registering the reconstructed 3D volumes by optimizing different metrics, such as mutual information (Coselmon et al 2004) or squared distance (Handels et al 2006, Ehrhardt et al 2007). To speed up the process, it is also possible to register the $3 \mathrm{D}$ image at maximum inspiration with the one at maximum expiration and interpolate the intermediate phases (Schreibmann et al 2006), but this procedure requires a respiratory signal before registration. Another option to reduce acquisition time and patient exposure is to obtain a high-quality acquisition to be used as a reference for the registration process of subsequent fast low-quality scans (McClelland et al 2006). The resulting deformation field enables the construction of a high-quality dynamic study by deforming the initial reference.

Finally, another approach involves modifying the reconstruction algorithm to include patient-specific motion models (Li et al 2006), time-varying magnification models (Crawford et al 1996) or dynamic particle models (Grangeat et al 2002). These approaches are usually complicated and may not yield appropriate results if the mathematical assumptions involved do not perfectly suit the problem.

Most of the literature mentioned above focuses on human imaging, but both prospective (Ford et al 2005, Badea et al 2004) and retrospective gating techniques (Hu et al 2004, 
Drangova et al 2007) have also been applied to image small animals with dedicated CT scanners. It is also possible to mechanically ventilate the animals to obtain triggered or breath-hold acquisitions (Namati et al 2006), but the technique becomes more cumbersome as intubation and animal breathing management are required to avoid atelectasis. Registration techniques have also been applied to animal lung imaging by exploiting a previously extracted breathing signal (Xu et al 2006).

In the present work, we implemented a fast, retrospective algorithm to extract the respiratory signal from CT projections in cone-beam geometry, thus avoiding the use of any additional respiratory monitoring instruments. The acquisition requires at least one breathing cycle per projection. We verified that, in micro-CT scanners based on flat-panel detectors with at least 8 frames $\mathrm{s}^{-1}$ acquisition rate, a respiratory signal can be generated from the sequence of frames acquired in each detector position by subtracting an averaged image from each of the original frames. The process is software based and totally automatic.

\section{Materials and methods}

\subsection{Algorithm proposed}

The method we propose is suitable for cone-beam micro-CT scanners equipped with flatpanel detectors and step-and-shoot multiframe acquisition protocols. With this protocol, the rotating gantry, to which the source and detector are attached, stops at each angular position and acquires several frames before rotating to the next angular position ('step-and-shoot'). The number of frames per position should cover at least one complete respiratory cycle, and the anaesthetized animals can breathe freely. The use of flat-panel detectors requires some pre-processing to correct the dark current effect, defective pixels and lines and to obtain the attenuation projections. This pre-processing is followed by the respiratory signal extraction and a phase-sorting algorithm. Finally, a 4D dataset is reconstructed. To ensure the robustness of our method regarding the different respiratory patterns observed for different types of anaesthesia, we performed an experiment with five test animals distributed into two groups anaesthetized with ketamine and isoflurane, respectively.

2.1.1. Respiratory signal extraction. From each set of frames, we obtain an average image which is then subtracted from each individual frame providing a set of difference images, $I_{d}$. In the area where there is lung movement, these difference images contain mainly positive values when they correspond to expiration and negative values for inspiration, while those pixels belonging to static areas have an almost zero value (figure 1).

A visual analysis of the histograms of these difference images indicates that both their mean value and symmetry change according to the respiratory phase (figure 2). We decided to calculate the first four central and non-central moments of these difference images and normalize the resulting signals to between -1 and 1 . This normalization obviates the scale factor; therefore, we calculated the moments by the following simplified expressions:

$$
\begin{aligned}
& \text { Non }- \text { central moments } \rightarrow \mu_{r}=\sum_{i=0}^{n} x_{i}^{r} \\
& \text { Central moments } \rightarrow \mu_{r}^{\prime}=\sum_{i=0}^{n}\left(x_{i}-\mu_{1}\right)^{r}
\end{aligned}
$$

where $r$ represents the order of the moment, $i$ is the pixel index, $x_{i}$ each pixel value and $n$ is the total number of pixels in the image. In equation (1.2), $\mu_{1}$ stands for the mean value, 


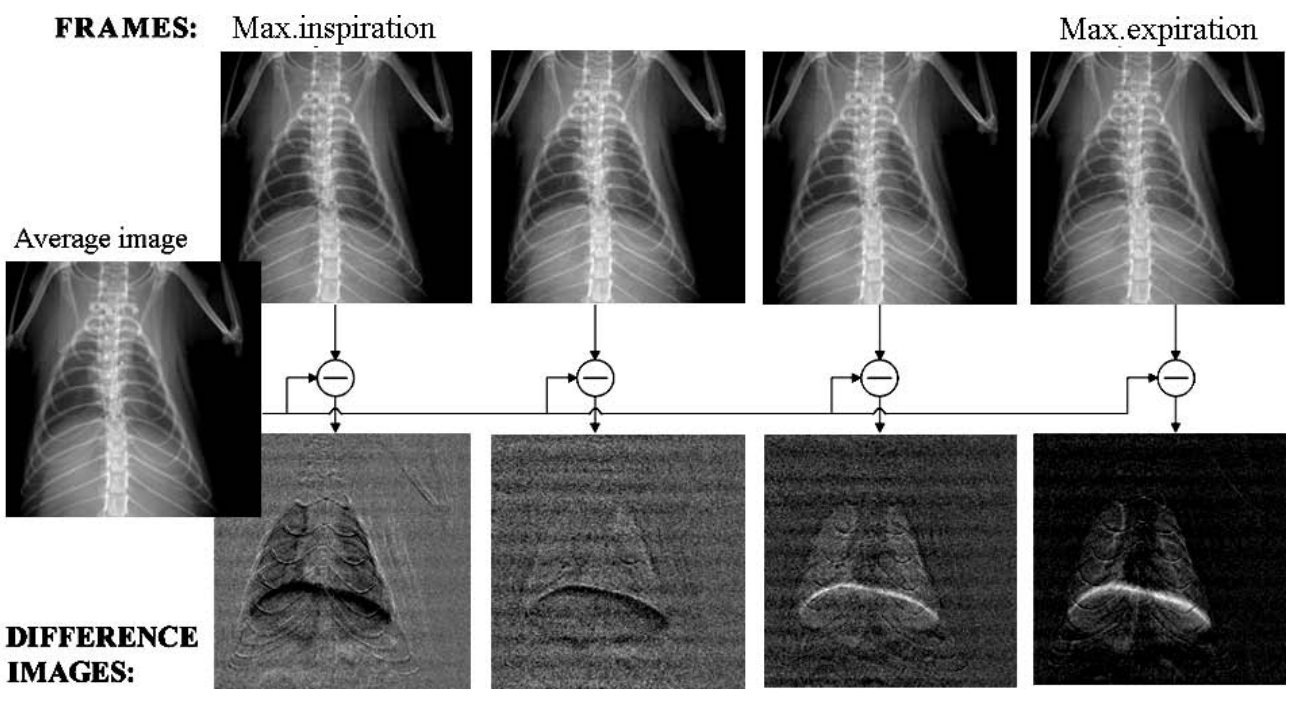

Figure 1. Upper row: original frames. Second row: difference images, where moving areas appear highlighted (zero value pixels are represented in grey, and positive and negative values are brighter and darker, respectively).

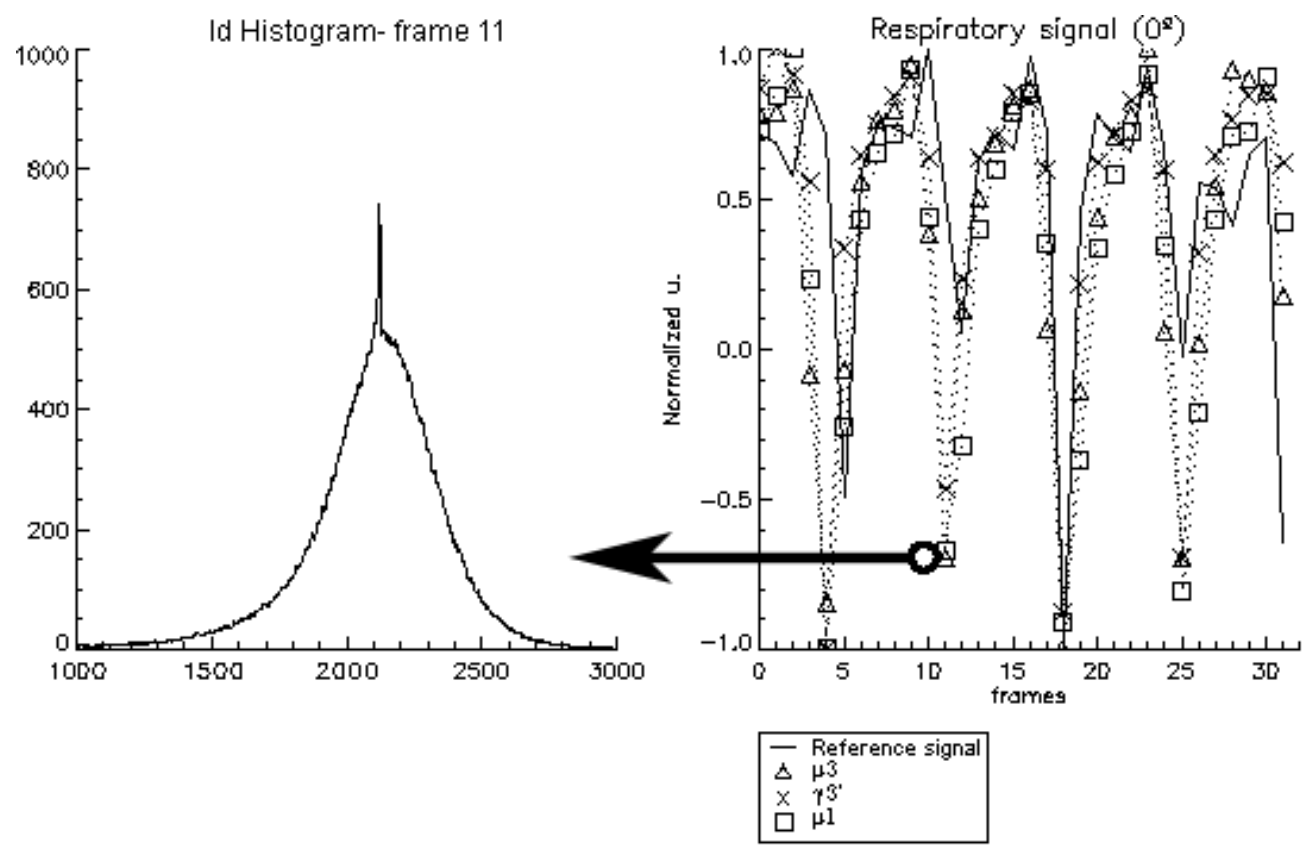

Figure 2. Left: histogram of one of the difference images, in this case at maximum inspiration (filtered by a boxcar of 10 pixels). Note the peak corresponding to the static areas, that is the grey pixels of $I_{d}$ (see figure 1). Right: the three respiratory signals extracted from the complete set of 32 frames and the reference signal.

that is, the first non-central moment. Even-order moments are always positive; therefore, the negative semicycle of the respiratory signal becomes rectified to positive and is no longer 
representative of the subject's respiration. As $\mu_{1}^{\prime}$ is always zero, we finally tested the signals provided by $\mu_{1}, \mu_{3}$ and $\gamma_{3}^{\prime}$. The latter represents the skewness (asymmetry) of the distribution and is calculated as the normalized $\mu_{3}^{\prime}$ :

$$
\gamma_{3}^{\prime}=\frac{\mu_{3}^{\prime}}{\left(\mu_{2}^{\prime}\right)^{\frac{3}{2}}}=\frac{\mu_{3}}{\sigma^{3}}=\sum_{i=1}^{n} \frac{\left(x_{i}-\mu_{1}\right)^{3}}{\sigma^{3}} .
$$

When using the multiframe 'step-and-shoot' acquisition protocol described above, we obtain one segment of respiratory signal per angular position of the rotating gantry (in our case 360 non-contiguous signal segments, as each step of the gantry is $1^{\circ}$ ). The number of samples in each segment of respiratory signal corresponds to the number of frames acquired at that specific angle, 32 in our case. Therefore, as we obtain one respiratory sample from each frame acquired, our respiratory signals become sampled at the frame acquisition rate (in our case 8 frames $\mathrm{s}^{-1}, 8 \mathrm{~Hz}$ ).

The three curves corresponding to $\mu_{1}, \mu_{3}$ and $\gamma_{3}^{\prime}$ were compared to an external reference respiratory signal recorded during acquisition. The curve showing better correlation with the reference, $\mu_{1}$, will be selected to represent the respiratory gating signal for our retrospective protocol (see validation and results).

2.1.2. Phase sorting and dynamic reconstruction. To generate a dynamic study from our raw data, we divided the amplitude of the breathing cycle into four different bins, each one corresponding to a different respiratory phase. This approach was preferred over phase sorting, since it was previously demonstrated that it provides higher similarity between the frames assigned to each bin (Wink et al 2006).

Frames were sorted by detecting all the maxima and minima of each signal segment (32 samples) and calculating the median value of all minima and maxima and the range between them. Afterwards, to define the four bins, we established three thresholds, at $1 / 6,1 / 2$ and $5 / 6$ of the amplitude range, in order to allow the maxima median and minima median be the centroids of bins 1 and 4, respectively. Given this amplitude sorting method, the number of frames inside each bin is not constant and depends on how the animal is breathing at that moment. Usually expiration is longer than inspiration, and therefore bin 1 (see figure 3 ) will have more frames than bin 4. This effect is even stronger under inhaled anaesthesia.

Averaging the frames assigned to each bin resulted in a set of four representative projections per angle. Finally, we reconstructed each bin independently and obtained a dynamic study of the subject composed of four image volumes, one at end expiration, another at end inspiration and two intermediate phases (figure 3).

\subsection{Validation}

2.2.1. CT scanner and data acquisition. The scanner used to carry out the validation was the CT subsystem of an eXplore PET/CT system (General Electric Healthcare) (Vaquero et al 2008). This system uses cone-beam geometry, a flat-panel detector and an x-ray source which offers up to $50 \mathrm{kV}$ and $1 \mathrm{~mA}$. Data are acquired with a frame grabber card. The field of view has a diameter of $71.5 \mathrm{~mm}$ and the measured resolution in reconstruction is $49 \mu \mathrm{m}$. Acquisition was based on a $1^{\circ}$ step-and-shoot protocol covering $360^{\circ}$, with multi-frame set-up to obtain 32 frames per gantry position at a speed of 8 frames $\mathrm{s}^{-1}$. All the acquisitions were obtained with a pixel binning of 4 and the resulting image size was $512 \times 512$ pixels. X-ray source parameters were $50 \mathrm{kV}$ and $200 \mu \mathrm{A}$ and the total study duration was about $25 \mathrm{~min}$. The total dose delivered was $0.48 \mathrm{~Gy}$. 


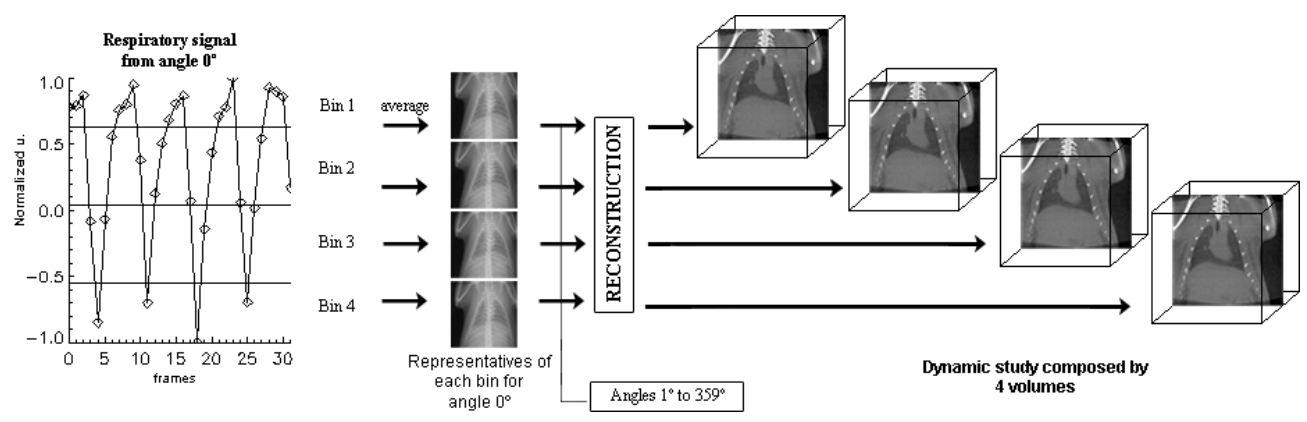

Figure 3. Description of the image sorting algorithm (for image details see figure 5). Left panel: respiratory signal obtained from one angular position and its binning classification. Each bin or amplitude group is averaged, resulting in one representative projection from each bin per gantry position. Then, all representatives of each bin (from angles $0^{\circ}$ to $360^{\circ}$ ) are used to reconstruct each respiratory phase separately. Finally, four image volumes are obtained, one per bin, which compose the desired dynamic study.

The physiological signal used as a reference for validation was obtained during the acquisition by means of a pneumatic pillow placed under the subject's body connected to an air-pressure sensor. It was then amplified, digitalized and recorded with a general-purpose laptop. We simultaneously extracted and recorded a synchronism signal from the scanner which indicated the periods of frame acquisition and the periods of gantry rotation, when no frames are acquired. Both signals were recorded at $44 \mathrm{kHz}$ using an A/D converter with a USB interface (Edirol UA-1A, Roland Corporation) and resampled to $8 \mathrm{~Hz}$. The synchronism signal allowed us to build a common time axis for the continuous reference signal recorded and the segments of respiratory signal extracted from the projections themselves.

Once we calculated the three candidate signals in the proper time axis, we compared each of them with the reference signal recorded during acquisition. For this purpose, we calculated the correlation coefficients between each of them and the reference signal. The signal selected for our retrospective protocol was that with highest correlation coefficient, namely, the mean or first non-central moment (see results).

Finally, after the binning process, all the 3D reconstructions were performed by means of a Feldkamp algorithm implemented in C.

2.2.2. Test animals. The specimens used for the validation were five randomly chosen 10 week-old adult Wistar rats weighing $300 \mathrm{~g}$ on average. Rats 2 and 3 were females and the rest were males. Rats 1-3 were anaesthetized with isoflurane at 3\% during induction and $1.5 \%$ for maintenance, whereas rats 4 and 5 were anaesthetized with an intra-peritoneal injection of ketamine (a mixture of $85 \mathrm{mg} \mathrm{kg}^{-1}$ ketamine and $2.5 \mathrm{mg} \mathrm{kg}^{-1}$ chlorpromazine).

2.2.3. Measurements. To assess the effectiveness our 4D-CT retrospective gating protocol we measured the sharpness of the diaphragm-lung transition in the reconstructed non-gated volume and gated image volume corresponding to bin 1 (end expiration). We calculated the average slope from $10 \%$ to $90 \%$ height on five adjacent vertical profiles drawn at the diaphragm dome in coronal slices, following a procedure similar to that proposed by Moseley et al (2005). Profile data were smoothed (10-pixel boxcar uniform filter) before measuring to reduce noise. 

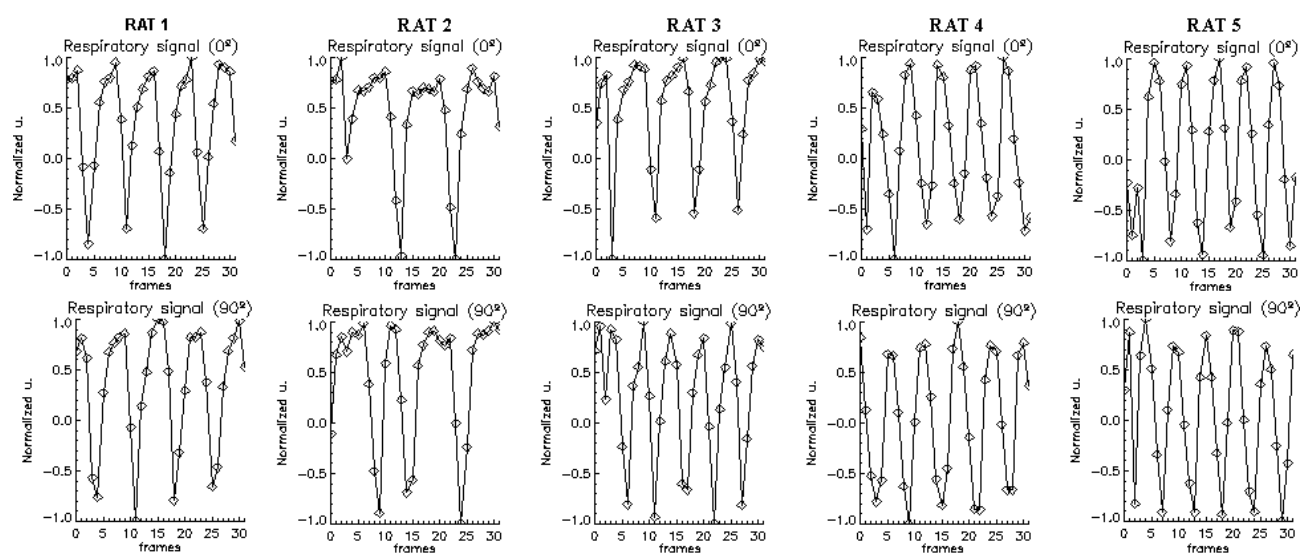

Figure 4. Breathing signals obtained for each animal at angles $0^{\circ}$ (from sagittal projection, acquisition time $=0 \mathrm{~s}$ ) and $90^{\circ}$ (from coronal projection, acquisition time $\sim 6 \mathrm{~min}$ ). Rats $1-3$ were anaesthetized with isoflurane and rats 4 and 5 with ketamine. Curves belonging to rats 4 and 5 show faster breathing and shorter end-expiration phases, as predicted. Due to the time lapse between the frames acquired at $0^{\circ}$ (upper row) and $90^{\circ}$ (lower row), the signal pattern changed considerably over time.

Table 1. Correlation coefficients of the three signals obtained from the distribution moments with the reference signal.

\begin{tabular}{lllllll}
\hline & & Rat 1 & Rat 2 & Rat 3 & Rat 4 & Rat 5 \\
\hline Non-central moments & $\mu_{1}$ (mean) & 0.506 & 0.481 & 0.428 & 0.524 & 0.356 \\
& $\mu_{3}$ & 0.436 & 0.426 & 0.370 & 0.473 & 0.307 \\
Central moments & $\gamma_{1}=\mu_{3}^{*} / \sigma^{3}$ (skewness) & 0.494 & 0.461 & 0.417 & 0.521 & 0.341 \\
\hline
\end{tabular}

\section{Results}

Figure 2 shows the histogram of a difference image, at maximum inspiration, within a set of 32 frames corresponding to rat 1 and projection angle $90^{\circ}$ (coronal).

We compared each of the candidate signals extracted from the CT projections with the reference provided by the pressure sensor by computing their respective correlation coefficients. Since all the candidate signals yielded very similar results (table 1), we selected the simplest one, the average pixel value. This procedure provides a respiratory signal that only differs in offset and scale from the one proposed by Hu et al (2004).

Figure 4 shows the respiratory signals obtained at different times during the studies. Note that, as each of the samples comes from analysing one of the frames acquired (subtracting the average image and then calculating the corresponding moment), all of them have the same sample rate $(8 \mathrm{~Hz})$ as our system frame acquisition frequency, 8 frames $\mathrm{s}^{-1}$. Each of the graphics corresponds to the 32 frames acquired from a particular axial angle, and therefore their duration in the horizontal axis is $4 \mathrm{~s}$. The differences in breathing patterns derived from the different anaesthesia procedures are remarkable: with ketamine (rats 4 and 5), the frames are evenly distributed into the four different bins, whereas isoflurane (rats 1-3) makes the end expiration longer and more stable, thus allowing the first bin to have better statistics and, subsequently, a better signal-to-noise ratio (SNR) (Hacker et al 2005). Even though all 


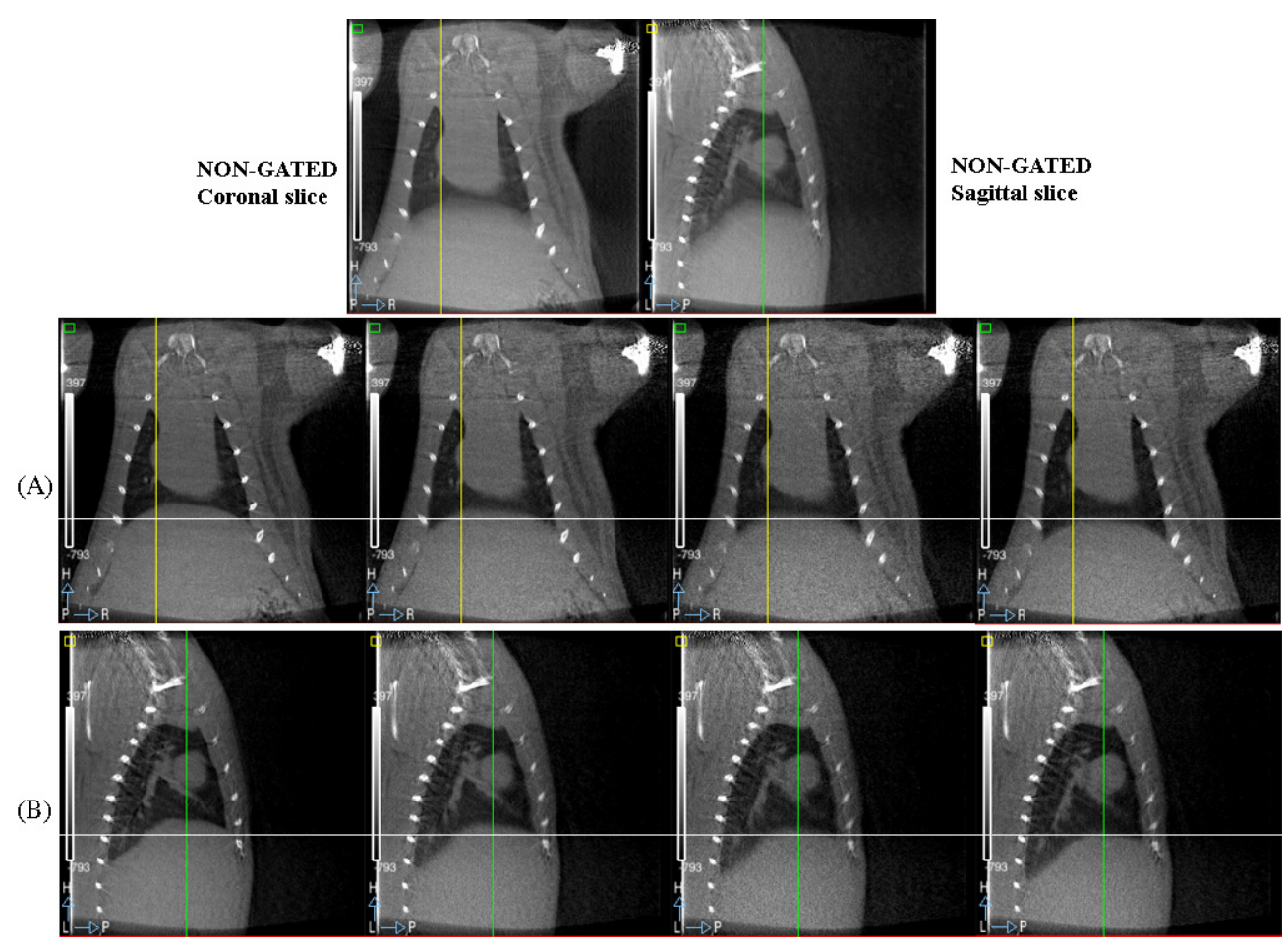

Figure 5. Upper row: non-gated study. (A) Coronal views of the dynamic study. From left to right: bin 1 (end expiration), bins 2-4 (end inspiration). (B) Sagittal views of the dynamic study. From left to right: bin 1 (end expiration), bins $2-4$ (end inspiration). The white lines drawn over the slices provide a common spatial reference to better appreciate the diaphragm motion. Scale range in all images: $[-793,397]$.

Table 2. Diaphragm dome slopes (Hounsfield units per pixel).

\begin{tabular}{lccl}
\hline & \multicolumn{2}{l}{ Slope (HU/pixel) } & \\
\cline { 2 - 3 } Animals & Gated & Non-gated & Slope improvement \\
\hline 1 & 12.8 & 10.4 & $23.2 \%$ \\
2 & 14.0 & 8.9 & $57.0 \%$ \\
3 & 12.3 & 6.7 & $84.8 \%$ \\
4 & 12.1 & 6.5 & $85.5 \%$ \\
5 & 9.7 & 6.4 & $53.1 \%$ \\
\hline
\end{tabular}

the signals appear to be quasi-periodic, we can appreciate a high variability of their period during the acquisition process, which is caused by physiological fluctuations of the respiratory rhythm. Note the change in breathing frequency between coronal (acquisition time $=0 \mathrm{~s}$ ) and sagittal projections (acquisition time approximately $6 \mathrm{~min}$ ) in rat 3.

When the resulting four volumes are assembled into a temporal sequence and played in cine mode, it is possible to distinguish the different positions of the diaphragm in each of the four cycle phases. An example of coronal and sagittal views is shown in figure 5 .

An average improvement of $60.7 \%$ was achieved in the slope of gated reconstructions. Individual values for all the study cases are shown in table 2. 


\section{Discussion}

The breathing signal extracted from the projections with our method matched the expected pattern, as inferred from the visual inspection of the corresponding projections. The differences observed between isoflurane and ketamine also agreed with the expected result and confirmed the robustness of our method in the presence of different breathing patterns. Furthermore, the gating signals obtained from the mean, third non-central moment and skewness curves were very similar to the reference signal, which supports the validity of either of them in a retrospective gating protocol. Reconstructed gated volumes at different phases showed a sharper diaphragm transition than that of the non-gated studies. The comparison between profiles in these studies allowed us to quantify the improvement in resolution.

It is difficult to compare our results with those of previous retrospective methods, because of the diversity of the metrics used and because many of them were only assessed with phantoms (Keall et al 2004, Pan et al 2004, Rietzel et al 2005, Vedam et al 2003, Sonke et al 2005). The work of Moseley et al who applied a similar metric involved a prospective breathhold technique with human patients, which prevented us from comparing results (Moseley et al 2005).

If we compare the gated and non-gated images we obtained, a decrement in signal-tonoise ratio (SNR) in the gated studies is observed. This is due to the separation of the information into different bins, thus reducing the number of averaged frames and increasing the Gaussian noise in the final images. However, this effect is present in every retrospective algorithm which divides the data into different groups. Moreover, the need for more than one cycle per slice increases the exposure, as compared with the single acquisition in prospective protocols. In our case, for example, to obtain a single volume of the same quality as our dynamic studies, a prospective method would deliver one quarter of the dose our method does. However, when a dynamic study is desired, prospective methods deliver the same dose as retrospective ones since additional acquisitions are necessary to obtain the remaining phases. One advantage of the retrospective approach is that a single acquisition offers information about all respiratory phases and enables a complete dynamic study without any external triggering system. Direct extraction of the respiratory signal from the cone-beam projections has an important advantage over external devices, since it not only avoids implementing the extra instrumentation, but it also makes the acquisition process much simpler and prevents skin contact artefacts. Furthermore, the proposed method is completely automatic, as it does not involve any user interaction (selecting regions of interest, thresholds or landmarks in the lungs).

It has been suggested that image registration techniques may result in smoother transitions between respiratory volumes at different phases (Xu et al 2006), but their computing burden is clearly higher than that of phase-sorting algorithms. Consequently, although in some cases the execution time and system requirements may make simpler solutions advisable, registration might be the best option whenever the spatial resolution is critical. Nevertheless, registration requires either a respiratory signal or a reference volume taken at breath-hold, which is usually hard to obtain in animal imaging.

A possible limitation to the precision of our method may derive from the fact that we obtain the respiratory signal by processing the whole image instead of making use of ROIs focused on the thorax (Hu et al 2004). Nevertheless, in the present work, we made no further study of this issue.

Another limitation of our work might be that we could not measure the blurring reduction in the airways themselves. But, given that the diaphragm is the structure which shows higher 
displacement due to respiratory motion, the improvement achieved at the diaphragm dome is representative for the effectiveness of our method.

In addition to these limitations, it is necessary to point out that we did not separate the respiratory phases themselves, as with the amplitude sorting method bins 2 and 3 include information about both inspiration and expiration. Anyway, the purpose of this work was to obtain sharper edges by averaging frames with the diaphragm in the same position, without considering if it is moving up or down.

The main advantage of the method proposed is its simplicity, which makes it fast and potentially able to perform phase sorting in real time, simultaneously with the acquisition. However, further research is warranted on the combination of our technique with image registration when pursuing better quality transitions.

In summary, we successfully developed an automatic and fast respiratory gating technique using a retrospective approach to provide both dynamic studies throughout the respiratory cycle and image blurring reduction, demonstrated in the quality of the diaphragm-lung transition.

\section{Acknowledgements}

The authors would like to thank Alexandra de Francisco for assistance with the study animals and Marina Benito for her help with documentation. This project was supported by the CENIT Programme (Ministerio de Industria), CIBER CB07/09/0031 (Ministerio de Sanidad y Consumo) and TEC2007-64731/TCM (Ministerio de Educación y Ciencia).

\section{References}

Badea C, Hedlund L W and Johnson G A 2004 Micro-CT with respiratory and cardiac gating Med. Phys. 31 3324-9 Beddar A S, Kainz K, Briere T M, Tsunashima Y, Pan T, Prado K, Mohan R, Gillin M and Krishnan S 2007 Correlation between internal fiducial tumor motion and external marker motion for liver tumors imaged with 4D-CT Int. J. Radiat. Oncol. Biol. Phys. 67 630-8

Coselmon M M, Balter J M, McShan D L and Kessler M L 2004 Mutual information based CT registration of the lung at exhale and inhale breathing states using thin-plate splines Med. Phys. 31 2942-8

Crawford C R, King K F, Ritchie C J and Godwin J D 1996 Respiratory compensation in projection imaging using a magnification and displacement model IEEE Trans. Med. Imaging 15 327-32

Damen E, Wolthaus J, Herk M V, Sonke J, Remeijer P, Zijp L, Belderbos J, Jaeger K D, Lebesque J and Boersma L 2004 Image-guided radiotherapy for lung cancer: respiration-correlated (cone-beam) CT to verify tumor position and motion characteristics during treatment delivery Int. J. Radiat. Oncol. Biol. Phys. $60 \mathrm{~S} 198$

Drangova M, Ford N L, Detombe S A, Wheatley A R and Holdsworth D W 2007 Fast retrospectively gated quantitative four-dimensional (4D) cardiac micro computed tomography imaging of free-breathing mice Invest. Radiol. 42 85-94

Ehrhardt J, Werner R, Saring D, Frenzel T, Lu W, Low D and Handels H 2007 An optical flow based method for improved reconstruction of 4D CT data sets acquired during free breathing Med. Phys. 34 711-21

Ford E C, Mageras G S, Yorke E and Ling C C 2003 Respiration-correlated spiral CT: a method of measuring respiratory-induced anatomic motion for radiation treatment planning Med. Phys. 30 88-97

Ford N L, Nikolov H N, Norley C J, Thornton M M, Foster P J, Drangova M and Holdsworth D W 2005 Prospective respiratory-gated micro-CT of free breathing rodents Med. Phys. 32 2888-98

Ford N L, Wheatley A R, Holdsworth D W and Drangova M 2007 Optimization of a retrospective technique for respiratory-gated high speed micro-CT of free-breathing rodents Phys. Med. Biol. 52 5749-69

Grangeat P, Koenig A, Rodet T and Bonnet S 2002 Theoretical framework for a dynamic cone-beam reconstruction algorithm based on a dynamic particle model Phys. Med. Biol. 47 2611-25

Guerrero T, Sanders K, Castillo E, Zhang Y, Bidaut L, Pan T and Komaki R 2006 Dynamic ventilation imaging from four-dimensional computed tomography Phys. Med. Biol. 51 777-91 
Hacker S O, White C E and Black I H 2005 A comparison of target-controlled infusion versus volatile inhalant anesthesia for heart rate, respiratory rate, and recovery time in a rat model Contemp. Top. Lab. Anim. Sci. 44 $7-12$

Handels H, Werner R, Frenzel T, Saring D, Lu W, Low D and Ehrhardt J 2006 Generation of 4D CT image data and analysis of lung tumour mobility during the breathing cycle Stud. Health Technol. Inform. 124 977-82

Hardouin S N and Nagy A 2000 Mouse models for human disease Clin. Genet. 57 237-44

Hu J, Haworth S T, Molthen R C and Dawson C A 2004 Dynamic small animal lung imaging via a postacquisition respiratory gating technique using micro-cone beam computed tomography Acad. Radiol. 11 961-70

Jose B, Chu A M, Sharma S C, Tobin D A and Scott R M 1983 Computed tomography and radiotherapy in the treatment of cancer J. Surg. Oncol. 23 83-92

Keall P J, Starkschall G, Shukla H, Forster K M, Ortiz V, Stevens C W, Vedam S S, George R, Guerrero T and Mohan R 2004 Acquiring 4D thoracic CT scans using a multislice helical method Phys. Med. Biol. 49 2053-67

Kleshneva T, Muzik J and Alber M 2006 An algorithm for automatic determination of the respiratory phases in four-dimensional computed tomography Phys. Med. Biol. 51 N269-76

Koch N, Liu H H, Starkschall G, Jacobson M, Forster K, Liao Z, Komaki R and Stevens C W 2004 Evaluation of internal lung motion for respiratory-gated radiotherapy using MRI: Part I. Correlating internal lung motion with skin fiducial motion Int. J. Radiat. Oncol. Biol. Phys. 60 1459-72

Li T, Schreibmann E, Yang Y and Xing L 2006 Motion correction for improved target localization with on-board cone-beam computed tomography Phys. Med. Biol. 51 253-67

Li T and Xing L 2007 Optimizing 4D cone-beam CT acquisition protocol for external beam radiotherapy Int. J. Radiat. Oncol. Biol. Phys. 67 1211-9

Low D A et al 2003 A method for the reconstruction of four-dimensional synchronized CT scans acquired during free breathing Med. Phys. 30 1254-63

Lu W, Low D A, Parikh P J, Nystrom M M, El Naqa I M, Wahab S H, Handoko M, Fooshee D and Bradley J D 2005a Comparison of spirometry and abdominal height as four-dimensional computed tomography metrics in lung Med. Phys. 32 2351-7

Lu W et al 2005b Quantitation of the reconstruction quality of a four-dimensional computed tomography process for lung cancer patients Med. Phys. 32 890-901

McClelland J R, Blackall J M, Tarte S, Chandler A C, Hughes S, Ahmad S, Landau D B and Hawkes D J 2006 A continuous 4D motion model from multiple respiratory cycles for use in lung radiotherapy Med. Phys. 33 3348-58

Mira J G, Fullerton G D, Ezekiel J and Potter J L 1982 Evaluation of computed tomography numbers for treatment planning of lung cancer Int. J. Radiat. Oncol. Biol. Phys. $81625-8$

Moseley D J, Hawkins M, Eccles C, Euler C, White E A, Bissonnette J, Dawson L A and Jaffray D A 2005 Respiratory gated cone-beam CT volumetric imaging for external beam radiotherapy Int. J. Radiat. Oncol. Biol. Phys. 63 S27-8

Namati E, Chon D, Thiesse J, Hoffman E A, de Ryk J, Ross A and McLennan G 2006 In vivo micro-CT lung imaging via a computer-controlled intermittent iso-pressure breath hold (IIBH) technique Phys. Med. Biol. 51 6061-75

Pan T, Lee T Y, Rietzel E and Chen G T 2004 4D-CT imaging of a volume influenced by respiratory motion on multi-slice CT Med. Phys. 31 333-40

Rietzel E and Chen G T 2006 Improving retrospective sorting of 4D computed tomography data Med. Phys. 33 377-9

Rietzel E, Pan T and Chen G T 2005 Four-dimensional computed tomography: image formation and clinical protocol Med. Phys. 32 874-89

Rit S, Sarrut D and Ginestet C 2005 Respiratory signal extraction for 4D CT imaging of the thorax from cone-beam CT projections Int. Conf. Med. Image Comput. Comput. Assist. Interv. 8 556-63

Schreibmann E, Chen G T and Xing L 2006 Image interpolation in 4D CT using a B Spline deformable registration model Int. J. Radiat. Oncol. Biol. Phys. $641537-50$

Simon L, Giraud P, Servois V and Rosenwald J C 2005 Lung volume assessment for a cross-comparison of two breathing-adapted techniques in radiotherapy Int. J. Radiat. Oncol. Biol. Phys. 63 602-9

Sonke J J, Zijp L, Remeijer P and van Herk M 2005 Respiratory correlated cone beam CT Med. Phys. 32 1176-86

Tanaka R, Sanada S, Kobayashi T, Suzuki M, Matsui T and Matsui O 2006 Computerized methods for determining respiratory phase on dynamic chest radiographs obtained by a dynamic flat-panel detector (FPD) system $J$. Digit. Imaging $1941-51$

Underberg R W, Lagerwaard F J, Cuijpers J P, Slotman B J, van Sornsen de Koste J R and Senan S 2004 Fourdimensional CT scans for treatment planning in stereotactic radiotherapy for stage I lung cancer Int. J. Radiat. Oncol. Biol. Phys. 60 1283-90

Vaquero J J, Redondo S, Lage E, Abella M, Sisniega A, Tapias G, Soto-Montenegro M L and Desco M 2008 Assessment of a new high-performance small-animal x-ray tomograph IEEE Trans. Nucl. Sci. 55 898-905 
Vedam S S, Keall P J, Kini V R, Mostafavi H, Shukla H P and Mohan R 2003 Acquiring a four-dimensional computed tomography dataset using an external respiratory signal Phys. Med. Biol. 48 45-62

Wink N, Panknin C and Solberg T D 2006 Phase versus amplitude sorting of 4D-CT data J. Appl. Clin. Med. Phys. 7 77-85

Xu S, Taylor R H, Fichtinger G and Cleary K 2006 Lung deformation estimation and four-dimensional CT lung reconstruction Acad. Radiol. 13 1082-92

Zeng R, Fessler J A and Balter J M 2005 Estimating 3D respiratory motion from orbiting views Nucl. Sci. Symp. Conf. Record, 2005 IEEE pp 2399-403

Zijp L, Sonke J J and Herk M V 2004 Extraction of respiratory signal from sequential thorax cone-beam X-ray images Proc. 14th ICCR (Seoul, Korea) pp 507-9 